\title{
A NOTE ON AIG- CLOSURE AND AIG- INTERIOR IN IDEAL TOPOLOGICAL SPACES
}

\author{
D. Vinodhini ${ }^{\mathrm{a}}$, M. Karpagadevi ${ }^{\mathrm{b}}$, L. Senthil Kumar ${ }^{\mathrm{c}}$, and S.Santhiya ${ }^{\mathrm{d}}$
}

A, Department Of Mathematics, Amrita School of Agricultural Sciences, Coimbatore, Tamilnadu-642 109, India

${ }^{b}$ Department of mathematics, Sri g. V. G. Vishalakshi College for Women, Udumalpet, Tamilnadu-642 128, india

cDepartent of Mathematics, Dr. Mahalingam College of Engineering and Technology, Pollachi,Tamil Nadu -642130, india,

'Department of Mathematics, Sri Krishna College of Technology, Coimbatore, Tamil Nadu-641 008, India.

Article History: Received: 11 January 2021; Accepted: 27 February 2021; Published online: 5 April 2021

\begin{abstract}
The concepts of $\alpha$ Ig- closure, $\alpha$ Ig- interior and $\alpha$ Ig- boundary of a subset of an ideal topological space $(\mathrm{X}, \tau, \mathrm{I})$ are introduced in this article. Some of their basic properties are proven. Furthermore, the relationships between these sets are investigated to get the best of them. Also, it is established that $\alpha$ Ig-closure is a Kuratowski closure operator on $(X, \tau, I)$ under certain conditions.
\end{abstract}

KEYWORDS. $\alpha$ Ig- closed set, $\alpha$ Ig- closure, $\alpha$ Ig- interior, $\alpha$ Ig- boundary.

\section{Introduction}

Topology is a branch of mathematics, which is believed that topological structure will be an important base for modification of knowledge extraction and processing. Kuratowski's closure complement theorem [1] has been a guiding source of topology. Peleg[2], while investigating the transitive closure of a binary relation, came across several closure operators which do not satisfy some of the four of Kuratowski's closure axioms, though their properties suffice to maintain "closure complement phenomenon". Similar kind of generalized closure operators are generated by the monotonic mappings introduced by A. Csaszar[3]. When several such operators are considered simultaneously and composed, the study of closure complement phenomenon becomes complicated and highly interesting.

The contributions of Hamlett and Jankovic[4-7] in ideal topological spaces initiated the generalization of some important properties in general topology via topological ideals. Kuratowski and Vaidhanathaswamy studied the notion of ideal topological spaces. Dontchev et al, Navaneethakrishnan et al, Jankovic et aletc., were investigated applications to various fields of ideal topology. The properties like decomposition of continuity, separation axioms, connectedness, compactness and resolvability [8-12] have been generalized using the concept of ideals in topological spaces. In [13], O. Njasted investigated the notion of $\alpha$-closed sets. By using $\alpha$-open sets, Mashhour et al.[14] defined and studied the concept of $\alpha$-closed sets, $\alpha$-closure of a set, $\alpha$-continuity and $\alpha$ closedness in topology. In the present note,we provide our investigations regarding all possible compositions of generalized closure operators and their corresponding interior operators in ideal topological spaces.

A topological space is a pair $(X, \tau)$ consisting of a set $X$ and family $\tau$ of subsets of $X$ satisfying the following conditions: a) $\phi \in$ and $X \in \tau \quad b) \tau$ is closed under arbitrary union c) $\tau$ is closed under finite intersection. The elements of $X$ are called points of the space, the subsets of $X$ belonging to $\tau$ are called open sets in the space, the complement of the subsets of $\mathrm{X}$ belonging to $\tau$ are called closed sets in the space. The $\tau$-closure of a subset $\mathrm{A} \subseteq$ $\mathrm{X}$ is denoted by $\mathrm{cl}(\mathrm{A})$ which is defined as the smallest closed subset of $\mathrm{X}$ which contains $\mathrm{A}$. The $\tau$-interior of a subset $\mathrm{A} \subseteq \mathrm{X}$ is denoted by $\operatorname{int}(\mathrm{A})$ which is defined as the union of all open subsets of $\mathrm{X}$ which contained in $\mathrm{A}$. Note that $A$ is open if and only if $A=\operatorname{int}(A)$. The boundary of a subset $A \subseteq X$ is denoted by $b(A)$ and is given by

$\mathrm{b}(\mathrm{A})=\operatorname{cl}(\mathrm{A}) \quad \operatorname{int}(\mathrm{A})$.

An ideal I on a set $\mathrm{X}$ is a nonempty collection of subsets of $\mathrm{X}$ which satisfies:

a) $\mathrm{A} \in \mathrm{I}$ and $\mathrm{B} \subseteq \mathrm{A}$ implies that $\mathrm{B} \in \mathrm{I}$ and

b) $\mathrm{A} \in \mathrm{I}, \mathrm{B} \in \mathrm{I}$ implies that $\mathrm{A} \cup \mathrm{B} \in \mathrm{I}$.

An ideal topological space is a topological space $(X, \tau)$ with an ideal I on $X$ and it is denoted by $(X, \tau, I)$. Given a topological space $(X, \tau)$ with an ideal $I$ on $X$ and if $\rho(X)$ is the set of all subsets of $X$, a set operator $(*): \rho(X) \rightarrow \rho(X)$, is called a local function [15] of A with respect to $\tau$ and $I$, is defined as follows: for $A \subseteq X$, $A^{*}(X, I)=\{x \in U \cap A \notin I$ for every $U \in \tau(x)$ where $\tau(x)=\{U \in \tau(x) / x \in U\}\}$. A Kuratowski closure operator $\mathrm{cl}^{*}(\mathrm{~A})=\mathrm{A} \cup \mathrm{A}^{*}(\mathrm{I}, \tau)$.

Definition 1.1.[17]Let $(X, \tau)$ be a topological space and I be an ideal on X. A subset A of X is said to be $\alpha$-Ideal generalized closed set ( $\alpha$ Ig closed set) if $A^{*} \subseteq U$ whenever $A \subseteq U$ and $U$ is $\alpha$-open. The complement of $\alpha$ Ig closed set is called $\alpha$-Ideal generalized open set $(\alpha \operatorname{Ig}$ open set)

Definition 1.2.[18]Let $(X, \tau, I)$ be an ideal topological space and $x \in A \subseteq X$. Then $A$ is said to be an I $\alpha$ neighborhood of $\mathrm{x}$, if there exist an I $\alpha$-open set $\mathrm{U}$ such that $\mathrm{x} \in \mathrm{U} \subseteq \mathrm{A}$, and simply write as $\mathrm{I} \alpha \mathrm{N}(\mathrm{x})$. If $\mathrm{A}$ is $\mathrm{I} \alpha$ open set then it is I $\alpha$-open neighbourhood for any element $\mathrm{x} \in \mathrm{A}$. 
Definition 1.3.[18]Let $(X, \tau, \mathrm{I})$ be an ideal topological space and $\mathrm{x} \in \mathrm{A} \subseteq \mathrm{X}$. Then $\mathrm{x}$ is said to be an I $\alpha$-interior point of A if A contain an I $\alpha$-open neighborhood set for $\mathrm{x}$. The set of all I $\alpha$ - interior points of A is called I $\alpha$ interior set and simply is denoted by $\operatorname{I} \alpha$-int(A).

Definition 1.4.[18] Let $(X, \tau, I)$ be an ideal topological space and $A \subseteq X . x \in X$ is said to be an I $\alpha$-boundary point of $A$ if for every I $\alpha$-open neighborhood set for $x$ satisfies that the intersection with $A$ and $A^{c}$ is nonempty set. The set of all I $\alpha$-boundary points of A is called I $\alpha$-boundary set of A and simply is denoted by I $\alpha-b(A)$.

\section{2. $\alpha$ Ig-CLOSURE}

Definition 2.1. For every set $\mathrm{F} \subseteq(\mathrm{X}, \tau, \mathrm{I}), \alpha \mathrm{Ig}$-closure of $\mathrm{F}$ is defined as the intersection of all $\alpha \mathrm{Ig}$-closed sets containing F.(i.e.) $\alpha \operatorname{Ig}-\mathrm{cl}(\mathrm{F})=\cap\{\mathrm{A}: \mathrm{F} \subseteq \mathrm{A}, \mathrm{A} \in \alpha \operatorname{Ig}$-cl(X, $\tau, \mathrm{I})\}$.

Example 2.2.Let $X=\{a, b, c\}$ with topology $\tau=\{\phi,\{a\},\{b, c\}, X\}$ and $I=\{\phi,\{c\}\}$. Then $\alpha$ Ig- closure of $\{b\}$ is given as $\alpha \operatorname{Ig}-c l\{b\}=\cap\{\{b\},\{a, b\}, X /$ where $\{b\},\{a, b\}, X$ are $\alpha \operatorname{Ig}$ - closed set $\}$.

Theorem 2.3. If $\alpha \operatorname{Ig}-\operatorname{cl}(X, \tau, I)$ is closed under finite union, then $\alpha \operatorname{Ig}$-closure is a Kuratowski operator on $(X, \tau$, I) .

Proof. (i) $\alpha \operatorname{Ig}-\operatorname{cl}(\phi)=\phi$ and $\alpha \operatorname{Ig}-c 1(X)=X$ also $\mathrm{A} \subseteq \alpha \operatorname{Ig}-\operatorname{cl}(\mathrm{A})$.

(ii) Suppose that $E$ and $F$ are two subsets of $X$, then $\alpha \operatorname{Ig}-c l(E) \subseteq \alpha \operatorname{Ig}-c l(E \cup F)$ and $\alpha \operatorname{Ig}$-cl(F) $\subseteq \alpha I g-c l(E \cup F)$. Hence $\alpha \operatorname{Ig}$-cl(E) $\cup \alpha \operatorname{Ig}-c l(F) \subseteq \alpha \operatorname{Ig}-c l(E \cup F)$. If $x \notin \alpha \operatorname{Ig}-c l(E) \cup \alpha \operatorname{Ig}$-cl(F), then there exists $A, B \in \alpha \operatorname{Ig}$-cl(X, $\tau$, I) such that $\mathrm{E} \subseteq \mathrm{A}, \mathrm{x} \notin \mathrm{A}, \mathrm{F} \subseteq \mathrm{B}$ and $\mathrm{x} \notin \mathrm{B}$. Hence $\mathrm{E} \cup \mathrm{F} \subseteq \mathrm{A} \cup \mathrm{B}$ and $\mathrm{x} \notin \mathrm{A} \cup \mathrm{B}$. By hypothesis, $\mathrm{A} \cup \mathrm{B}$ is $\alpha \operatorname{Ig}$-closed. Thus, $\mathrm{x} \notin \alpha \operatorname{Ig}$-cl $(\mathrm{A} \cup \mathrm{B})$ implies that, $\mathrm{x} \notin \alpha \operatorname{Ig}$-cl $(\mathrm{E} \cup \mathrm{F})$. Hence $\alpha \operatorname{Ig}$-cl( $\mathrm{E} \cup \mathrm{F}) \subseteq \alpha \operatorname{Ig}$-cl( $\mathrm{E}) \cup \alpha \operatorname{Ig}$ $\operatorname{cl}(\mathrm{F})$. Thus, $\alpha \operatorname{Ig}-\mathrm{cl}(\mathrm{E} \cup \mathrm{F})=\alpha \operatorname{Ig}-\mathrm{cl}(\mathrm{E}) \cup \alpha \operatorname{Ig}-\mathrm{cl}(\mathrm{F})$.

(iii) Let $\mathrm{E} \subseteq \mathrm{X}$ and $\mathrm{A}$ be an $\alpha \mathrm{Ig}$-closed set containing E. Then by Definition 2.1., $\alpha \mathrm{Ig}$-cl(E) $\subseteq \mathrm{A}$ and $\alpha \mathrm{Ig}$-cl $(\alpha \mathrm{Ig}$ $\mathrm{cl}(\mathrm{E})) \subseteq \mathrm{A}$. Since $\alpha \operatorname{Ig}-\mathrm{cl}(\alpha \operatorname{Ig}-\mathrm{cl}(\mathrm{E})) \subseteq \mathrm{A}, \alpha \operatorname{Ig}-\operatorname{cl}(\alpha \operatorname{Ig}-\mathrm{cl}(\mathrm{E})) \subseteq \cap\{\mathrm{A}: \mathrm{E} \subseteq \mathrm{A}, \mathrm{A} \in \alpha \operatorname{Ig}-\mathrm{cl}(\mathrm{X}, \tau, \mathrm{I})\}$. Hence $(\alpha \operatorname{Ig}-$ $\operatorname{cl}(\mathrm{E})) \subset \alpha \operatorname{Ig}-\operatorname{cl}(\alpha \operatorname{Ig}-\mathrm{cl}(\mathrm{E}))$ and by Definition 2.1., $\alpha \operatorname{Ig}-\operatorname{cl}(\alpha \operatorname{Ig}-\mathrm{cl}(\mathrm{E})) \subset(\alpha \operatorname{Ig}-\mathrm{cl}(\mathrm{E}))$ implies that $(\alpha \operatorname{Ig}-\mathrm{cl}(\mathrm{E}))=\alpha \operatorname{Ig}-$ $\operatorname{cl}(\alpha \operatorname{Ig}-\mathrm{cl}(\mathrm{E}))$. Thus, $\alpha \mathrm{Ig}$-closure is a Kuratowski closure operator on $\mathrm{X}$.

Theorem 2.4. Let $A$ be the subset of the ideal topological spaces (X, $\tau, I)$. Then $x \in \alpha \operatorname{Ig}-c l(A)$ if and only if $A \cap$ $\mathrm{U} \neq \phi$ for every $\alpha \mathrm{Ig}$-open set $\mathrm{U}$ containing $\mathrm{X}$.

Proof. Suppose that $\mathrm{x} \in \alpha \mathrm{Ig}$-cl(A). Let $\mathrm{U}$ be an $\alpha \mathrm{Ig}$-open set containing $\mathrm{x}$ such that $\mathrm{A} \cap \mathrm{U}=\phi$ and so $\mathrm{A} \subset \mathrm{X}$

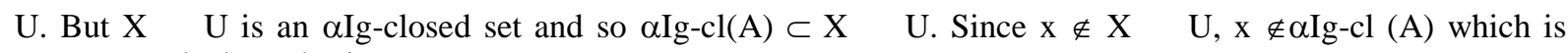
contrary to the hypothesis.

Conversely, suppose that every $\alpha$ Ig-open set of $X$ containing $x$ such that $A \cap U \neq \phi$. If $x \notin \alpha \operatorname{Ig}$-cl( $A$ ), then there exists an $\alpha I g$-closed set $F$ of $X$ such that $A \subseteq F$ and $x \notin F$. Hence $x \in X \quad F$ and $X \quad F$ is an $\alpha I g$-open set containing $\mathrm{x}$. Thus, $(\mathrm{X} \quad \mathrm{F}) \cap \mathrm{A}=\phi$ which is a contradiction.

\section{Ig-INTERIOR}

Definition 3.1. For every set $\mathrm{A} \subseteq \mathrm{X}, \alpha \mathrm{Ig}$-interior of $\mathrm{A}$ is defined to be the union of all $\alpha \mathrm{Ig}$-open sets contained in A.(i.e.) $\alpha \operatorname{Ig}$-int $(A)=\cup\{U: U \subseteq A, U \in \alpha \operatorname{Ig}$-int $(X, \tau, I)\}$.

Example 3.2.Let $X=\{a, b, c\}$ with topology $\tau=\{\phi,\{a\},\{a, c\}, X\}$ and $I=\{\phi,\{b\}\}$. Then $\alpha \operatorname{Ig}$ - interior of $\{a$, $\mathrm{b}\}$ is given as $\alpha \operatorname{Ig}$-int $\{\mathrm{a}, \mathrm{b}\}=\cup\{\{\mathrm{b}\},\{\mathrm{a}, \mathrm{b}\} /\{\mathrm{b}\},\{\mathrm{a}, \mathrm{b}\}$ are $\alpha \operatorname{Ig}$-open set $\}$

Theorem 3.3. Let $(X, \tau, I)$ be an ideal topological space and A be a subset of $X$, then the following statements are true.

(i) $\mathrm{X}-(\alpha \operatorname{Ig}-\operatorname{int}(\mathrm{A}))=\alpha \operatorname{Ig}-\operatorname{int}(\mathrm{X}-\mathrm{A})$.

(ii) $\alpha \operatorname{Ig}$-int $(\mathrm{A})=\mathrm{X}-(\alpha \operatorname{Ig}-\operatorname{int}(\mathrm{X}-\mathrm{A}))$.

(iii) $\alpha \operatorname{Ig}-\operatorname{cl}(\mathrm{A})=\mathrm{X}-(\alpha \operatorname{Ig}-i n t(\mathrm{~A}))$.

Proof. (i) Let $x \in X-(\alpha \operatorname{Ig}$-int(A)). Then $\notin \notin(\alpha \operatorname{Ig}$-int(A)). That is, every $\alpha \operatorname{Ig}$-open set $U$ containing $x$ is such that $\mathrm{U}$ is not a proper subset of $\mathrm{A}$. Thus, $\mathrm{U} \cap(\mathrm{X}-\mathrm{A}) \neq \phi$, for every $\alpha \mathrm{Ig}$-open set $\mathrm{U}$ containing $\mathrm{x}$. Hence, $\mathrm{x} \in \alpha \mathrm{Ig}-$ $\operatorname{int}(X-A)$ and so $X-(\alpha \operatorname{Ig}-i n t(A)) \subseteq \alpha \operatorname{Ig}$-int $(X-A)$. Conversely, suppose $x \in \alpha \operatorname{Ig}$-int $(X-A)$. Then, $U \cap(X$ A) $\neq \phi$, for every $\alpha \operatorname{Ig}$-open set $U$ containing $x$. By Definition3.1., $x \notin(\alpha \operatorname{Ig}$-int $(A))$. Thus, $x \in X-(\alpha \operatorname{Ig}$-int $(A))$ and so $(\alpha \operatorname{Ig}-\operatorname{int}(X-A)) \subseteq X-(\alpha \operatorname{Ig}$-int $(A))$. Therefore, $X-(\alpha \operatorname{Ig}-i n t(A))=\alpha \operatorname{Ig}$-int $(X-A)$.

(ii) From (i), $X-(\alpha \operatorname{Ig}$-int $(A))=\alpha \operatorname{Ig}$-int $(X-A)$. By taking the complement on both sides, we get $(X-(X-\alpha I g-$ $\operatorname{int}(\mathrm{A})))=(\mathrm{X}-\alpha \operatorname{Ig}-\operatorname{int}(\mathrm{X}-\mathrm{A}))$ implies that $\alpha \operatorname{Ig}$-int $(\mathrm{A})=\mathrm{X}-(\alpha \operatorname{Ig}-\operatorname{int}(\mathrm{X}-\mathrm{A}))$.

(iv) From (i), $X-(\alpha \operatorname{Ig}-i n t(A)=\alpha \operatorname{Ig}$-int $(X-A)$. Replace $A$ by $X-A$, we get $X-(\alpha \operatorname{Ig}-i n t(X-A))=X-\alpha \operatorname{Ig}$ $\operatorname{int}((X-A))$ which implies that $\alpha \operatorname{Ig}-\operatorname{cl}(A)=X-(\alpha \operatorname{Ig}-\operatorname{int}(X-A))$.

\section{4. $\alpha$ Ig-BOUNDARY}


Definition 4.1. Consider an ideal topological space $(X, \tau, I)$. Let $A$ be any subset of $(X, \tau, I)$. Then, the $\alpha \operatorname{Ig}-$ boundary of $\mathrm{A}$ is defined as $\alpha \operatorname{Ig}-\mathrm{Bd}(\mathrm{A})=\alpha \operatorname{Ig}-\mathrm{cl}(\mathrm{A}) \cap \alpha \operatorname{Ig}-\mathrm{cl}(\mathrm{X}-\mathrm{A})$.

Theorem 4.2. For any sets $A$ and $B$ in ideal topological space $(X, \tau, I)$, the following conditions hold:

(i) $\alpha \operatorname{Ig}-\mathrm{Bd}(\mathrm{A})=\alpha \operatorname{Ig}-\mathrm{Bd}(\mathrm{X}-\mathrm{A})$.

(ii) $\alpha \operatorname{Ig}-\mathrm{Bd}(\mathrm{A}) \subseteq \alpha \operatorname{Ig}-\mathrm{cl}(\mathrm{A})$.

(iii) $\alpha \operatorname{Ig}-\mathrm{Bd}(\mathrm{A}) \subseteq \alpha \operatorname{Ig}-\mathrm{cl}(\mathrm{X}-\mathrm{A})$.

(iv) If $\mathrm{A}$ is an $\alpha \operatorname{Ig}$-closed set, then $\alpha \operatorname{Ig}-\mathrm{Bd}(\mathrm{A}) \subseteq \mathrm{A}$.

(v) If $\mathrm{A}$ is an $\alpha \mathrm{Ig}$-open set, then $\alpha \operatorname{Ig}-\mathrm{Bd}(\mathrm{A}) \subseteq \mathrm{X}-\mathrm{A}$.

(vi) Let $\mathrm{A} \subseteq \mathrm{B}$ and $\mathrm{B} \in \alpha \operatorname{Ig}-\mathrm{cl}(\mathrm{X}, \tau)$ (resp. $\mathrm{B} \in \alpha \operatorname{Ig}-\mathrm{O}(\mathrm{X}, \tau)$ ). Then, $\alpha \operatorname{Ig}-\mathrm{Bd}(\mathrm{A}) \subseteq \mathrm{B}$ (resp. $\left.\alpha \operatorname{Ig}-\mathrm{Bd}(\mathrm{A}) \subseteq \mathrm{B}^{\mathrm{c}}\right)$, where $\alpha \operatorname{Ig}-\operatorname{cl}(X, \tau)$ denotes the class of $\alpha \operatorname{Ig}$-closed (resp. $\alpha \operatorname{Ig}-\mathrm{O}(\mathrm{X}, \tau)$ denotes the class of $\alpha \operatorname{Ig}$-open) sets in $X$.

(vii) $\mathrm{X}-(\alpha \operatorname{Ig}-\mathrm{Bd}(\mathrm{A}))=\alpha \operatorname{Ig}$-int $(\mathrm{A}) \cup \alpha \operatorname{Ig}$-int $(\mathrm{X}-\mathrm{A})$.

Proof. (i) $\alpha \operatorname{Ig}-\mathrm{Bd}(\mathrm{A})=\alpha \operatorname{Ig}-\operatorname{cl}(\mathrm{A}) \cap \alpha \operatorname{Ig}-\mathrm{cl}(\mathrm{X}-\mathrm{A})=\alpha \operatorname{Ig}-\mathrm{cl}(\mathrm{X}-\mathrm{A}) \cap \alpha \operatorname{Ig}-\mathrm{Cl}(\mathrm{A})=\alpha \operatorname{Ig}-\mathrm{cl}(\mathrm{X}-\mathrm{A}) \cap(\mathrm{X}-\alpha \operatorname{Ig}-$ $\operatorname{cl}(\mathrm{X}-\mathrm{A}))=\alpha \operatorname{Ig}-\mathrm{Bd}(\mathrm{X}-\mathrm{A})$.

(ii) By definition 4.1, $\alpha \operatorname{Ig}-\mathrm{Bd}(\mathrm{A})=\alpha \operatorname{Ig}-\mathrm{cl}(\mathrm{A}) \cap \alpha \operatorname{Ig}-\mathrm{cl}(\mathrm{X}-\mathrm{A})$. Hence $\alpha \operatorname{Ig}-\mathrm{Bd}(\mathrm{A}) \subseteq \alpha \operatorname{Ig}-\mathrm{cl}(\mathrm{A})$.

(iii) By definition 4.1, $\alpha \operatorname{Ig}-\mathrm{Bd}(\mathrm{A})=\alpha \operatorname{Ig}-\mathrm{cl}(\mathrm{A}) \cap \alpha \operatorname{Ig}-\mathrm{cl}(\mathrm{X}-\mathrm{A})$. $T h u s \alpha \operatorname{Ig}-\mathrm{Bd}(\mathrm{A}) \subseteq \alpha \operatorname{Ig}-\mathrm{cl}(\mathrm{X}-\mathrm{A})$.

(iv) $\alpha \operatorname{Ig}-\mathrm{Bd}(\mathrm{A})=\alpha \operatorname{Ig}-\operatorname{cl}(\mathrm{A}) \cap \alpha \operatorname{Ig}-\operatorname{cl}(\mathrm{X}-\mathrm{A}) \subseteq \alpha \operatorname{Ig}-\operatorname{cl}(\mathrm{A})=\mathrm{A}$.

(v) If $\mathrm{A}$ is $\alpha \operatorname{Ig}$-open then, $\mathrm{X}-\mathrm{A}$ is $\alpha \operatorname{Ig}$-closed. By (iv), $\alpha \operatorname{Ig}-\mathrm{Bd}(\mathrm{X}-\mathrm{A}) \subseteq \mathrm{X}-\mathrm{A}$. Again by (i), $\alpha \operatorname{Ig}-\mathrm{Bd}(\mathrm{A}) \subseteq \mathrm{X}$ - A.

(vi) Since $\mathrm{A} \subseteq \mathrm{B}, \alpha \operatorname{Ig}-\mathrm{cl}(\mathrm{A}) \subseteq \alpha \operatorname{Ig}-\mathrm{cl}(\mathrm{B})$. Now, $\alpha \operatorname{Ig}-\mathrm{Bd}(\mathrm{A})=\alpha \operatorname{Ig}-\mathrm{cl}(\mathrm{A}) \cap \alpha \operatorname{Ig}-\mathrm{cl}(\mathrm{X}-\mathrm{A}) \subseteq \alpha \operatorname{Ig}-\mathrm{cl}(\mathrm{A}) \subseteq \alpha \operatorname{Ig}-\mathrm{cl}(\mathrm{B})$ $=\mathrm{B}$. Hence $\alpha \operatorname{Ig}-\mathrm{Bd}(\mathrm{A}) \subseteq \mathrm{B}$.

(vii) $\mathrm{X}-(\alpha \operatorname{Ig}-\mathrm{Bd}(\mathrm{A}))=\mathrm{X}-(\alpha \operatorname{Ig}-\mathrm{cl}(\mathrm{A}) \cap \alpha \operatorname{Ig}-\mathrm{cl}(\mathrm{X}-\mathrm{A}))=\mathrm{X}-(\alpha \operatorname{Ig}-\mathrm{cl}(\mathrm{A})) \cup \mathrm{X}-(\alpha \operatorname{Ig}-\operatorname{cl}(\mathrm{X}-\mathrm{A}))=(\alpha \operatorname{Ig}-\operatorname{int}(\mathrm{X}$ $-\mathrm{A})) \cup \mathrm{X}-(\alpha \operatorname{Ig}-\operatorname{int}(\mathrm{X}-\mathrm{A}))=\alpha \operatorname{Ig}-\operatorname{int}(\mathrm{X}-\mathrm{A})) \cup \alpha \operatorname{Ig}-\operatorname{int}(\mathrm{A})$.

Definition 4.3. Let $(X, \tau, I)$ be an ideal topological space and $A \subseteq X$. Then, $\alpha I g$-border of $A$ is defined as $\operatorname{b} \alpha \operatorname{Ig}(\mathrm{A})=\mathrm{A} \quad \alpha \operatorname{Ig}-\operatorname{int}(\mathrm{A})$.

Theorem 4.4. For a subset $A$ of an ideal topological space $(X, \tau, I)$, the following conditions hold:

(i) $\alpha \operatorname{Ig}-\mathrm{Bd}(\mathrm{A}) \subseteq \mathrm{Bd}(\mathrm{A})$, where $\mathrm{Bd}(\mathrm{A})$ denotes the boundary of $\mathrm{A}$.

(ii) $\alpha \operatorname{Ig}-c l(A)=\alpha \operatorname{Ig}-i n t(A) \cup \alpha \operatorname{Ig}-B d(A)$

(iii) $\alpha \operatorname{Ig}-\operatorname{int}(\mathrm{A}) \cap \alpha \operatorname{Ig}-\mathrm{Bd}(\mathrm{A})=\phi$

(iv) $b \alpha \operatorname{Ig}(\mathrm{A}) \subseteq \alpha \operatorname{Ig}-\mathrm{Bd}(\mathrm{A})$

(v) $\alpha \operatorname{Ig}-\mathrm{Bd}(\mathrm{int}(\mathrm{A})) \subseteq \alpha \operatorname{Ig}-\mathrm{Bd}(\mathrm{A})$

(vi) $\alpha \operatorname{Ig}-\mathrm{Bd}(\mathrm{cl}(\mathrm{A})) \subseteq \alpha \operatorname{Ig}-\mathrm{Bd}(\mathrm{A})$.

Proof. (i) $\alpha \operatorname{Ig}-\mathrm{Bd}(\mathrm{A})=\alpha \operatorname{Ig}-\mathrm{cl}(\mathrm{A}) \cap \alpha \operatorname{Ig}-\mathrm{cl}(\mathrm{X}-\mathrm{A}) \subseteq \operatorname{cl}(\mathrm{A}) \cap \operatorname{cl}(\mathrm{X}-\mathrm{A})=\mathrm{Bd}(\mathrm{A})$.

(ii) $\alpha \operatorname{Ig}-\operatorname{int}(\mathrm{A}) \cup \alpha \operatorname{Ig}-\mathrm{Bd}(\mathrm{A})=\alpha \operatorname{Ig}-\operatorname{int}(\mathrm{A}) \cup(\alpha \operatorname{Ig}-\mathrm{cl}(\mathrm{A}) \cap \alpha \operatorname{Ig}-\mathrm{cl}(\mathrm{X}-\mathrm{A}))=\alpha \operatorname{Ig}-\mathrm{cl}(\mathrm{A})$.

(iii) $\alpha \operatorname{Ig}$-int $(\mathrm{A}) \cap \alpha \operatorname{Ig}-\mathrm{Bd}(\mathrm{A})=\alpha \operatorname{Ig}-\operatorname{int}(\mathrm{A}) \cap(\alpha \operatorname{Ig}-\mathrm{cl}(\mathrm{A}) \cap \alpha \operatorname{Ig}-\mathrm{cl}(\mathrm{X}-\mathrm{A}))=\phi$.

(iv) b $\alpha \operatorname{Ig}(\mathrm{A})=\mathrm{A} \quad \alpha \operatorname{Ig}$-int(A) $\subseteq \alpha \operatorname{Ig}$-cl (A) $\quad \alpha \operatorname{Ig}$-int(A) $\subseteq \alpha \operatorname{Ig}$-cl (A) $\cap \mathrm{X}-(\alpha \operatorname{Ig}$-int(A) $\subseteq \alpha \operatorname{Ig}$-cl $(\mathrm{A}) \cap \alpha \operatorname{Ig}$ $\operatorname{cl}(\mathrm{X}-\mathrm{A})=\alpha \operatorname{Ig}-\mathrm{Bd}(\mathrm{A})$.

(v) $\alpha \operatorname{Ig}-\mathrm{Bd}(\operatorname{int}(\mathrm{A}))=\alpha \operatorname{Ig}-\mathrm{cl}(\operatorname{int}(\mathrm{A})) \cap \mathrm{X}-\alpha \operatorname{Ig}-\mathrm{cl}(\operatorname{int}(\mathrm{A})) \subseteq \alpha \operatorname{Ig}-\mathrm{cl}((\mathrm{A})) \cap \mathrm{X}-(\alpha \operatorname{Ig}$-int $(\mathrm{A})) \quad=\alpha \operatorname{Ig}-\mathrm{Bd}(\mathrm{A})$.

(vi) $\alpha \operatorname{Ig}-\mathrm{Bd}(\mathrm{Cl}(\mathrm{A}))=\alpha \operatorname{Ig}-\operatorname{cl}(\operatorname{cl}(\mathrm{A})) \cap \alpha \operatorname{Ig}-\operatorname{cl}(\operatorname{cl}(\mathrm{A})) \mathrm{c}=\alpha \operatorname{Ig}-\operatorname{cl}(\mathrm{A}) \cap(\alpha \operatorname{Ig}-\operatorname{int}(\operatorname{cl}(\mathrm{A}))) \subseteq \alpha \operatorname{Ig}-\operatorname{cl}(\mathrm{A}) \cap \mathrm{X}-(\alpha \operatorname{Ig}-$ $\operatorname{int}(\mathrm{A}))=\alpha \operatorname{Ig}-\mathrm{Bd}(\mathrm{A})$.

\section{CONCLUSION}

In this present work, we introduced the new concepts $\alpha$ Ig-closure, $\alpha$ Ig-interior and $\alpha$ Ig- boundary of a subset of an ideal topological space $(\mathrm{X}, \tau, \mathrm{I})$. Also we studied and proved the basic properties of these concepts

\section{REFERENCES}

1. C. Kuratowski, Sur l'operationA de l'analysissitus, Fund. Math. 3 (1922), 182-199.

2. D. Peleg, A Generalized closure and complement phenomenon, Discrete Math. 50 (1984), 285293

3. . Cs sz r, Generalized open sets, Acta Math. Hungar. 75 (1997), 65-87.

4. Bhuvaneswari, K., \&Ezhilarasi, A. (2014). On nano semi-generalized and nano generalized-semi closed sets in nano topological spaces. International Journal of Mathematics and Computer Applications Research, 4(3), 117-124.

5. T. R. Hamlett and D. Jankovic, Ideals in general topology, GeneralTopologyandApplications, (1988), 115-125. 
6. T.R.HamlettandD.Jankovic,Idealsin Topological Spacesand the Set Operatory," Bollettinodell'UnioneMatematicaItaliana, 7, (1990), 863-874.

7. D. Jankovic and T. R. Hamlett, New topologies from old via ideals, The American Mathematical Monthly, 97, (1990), 295-310.

8. Mohmmed, H. A., \&Anssari, O. M. H. (2016). current source 5, 9 \& 17 level by using main inverter and auxiliary inductor cells. BEST: International Journal of Management, Information Technology and Engineering (BEST: IJMITE), 4(4), 97-106.

9. D. Jankovic and T. R. Hamlett, Compatible extensions of ideals,BollettinodellaUnioneMatematicaItaliana, 7(6),(1992), 453-465.

10. G.Aslim,A.CaksuGulerandT.Noiri,On Decompositionsof Continuity and Some Weaker Forms of Continuity via Idealization,ActaMathematicaHungarica,109(3),(2005), 183-190.

11. Abd El-latif, A. M., \&Karatas, S. (2015). Supra b-open soft sets and supra b-soft continuity on soft topological spaces. Journal of Mathematics and Computer Applications Research, 5(1), 1-18.

12. F. G. Arenas, J. Dontchev, and M. L. Puertas, Idealization of some weak separation axioms, ActaMathematicaHungarica, 89(1-2),(2000),47-53.

13. J. Dontchev, M. Ganster and D. Rose, Ideal resolvability, TopologyandItsApplications,93(1), (1999), 1-16.

14. E.Ekici and T. Noiri, Connectedness in Ideal Topological Spaces,NoviSadJournalofMathematics,38(2), (2008), 65- 70.

15. AGARWAL, D., \& TYAGI, R. SOME PROPERTIES OF FIXED POINT THEOREM IN DMETRIC SPACE.

16. R.L.Newcomb, Topologies Whichare Compact Moduloan Ideal [Ph.D. dissertation], University of California at Santa Barbara, 1967.

17. O.Njastad, On some classes of nearly open sets, Pacific J.Math.,15, (1965),961-970

18. A. S. Mashhour, I.N.Hasanein and S.N. El-Deeb, $\alpha$-continuous and $\alpha$-open mappings, Acta Math. Hungar,41, (1983),213-218.

19. K. Kuratowski, Topology, Vol. I. New York: Academic Press, (1996).

20. Illesinghe, A., \&Palaniappan, S. TOWARDS PREDICTING CREDIT RISK IN SRI LANKA'S BANKING SECTOR.

21. S. G. Crossley and S. K. Hildebrand, Semi-closure, Texas J.Sci., 22, (1971), 99-112.

22. S. Maragathavally and D. Vinodhini, on $\alpha$ Generalized Closed Sets in Ideal Topological Spaces, IOSR- Journal of Mathematics, 10(2),(2014), 33- 38.

23. KASSI, K., \& MITTAL, A. MODIFIED SINGLE PHASE H-BRIDGE MULTI-LEVEL INVERTER TOPOLOGY WITH SPWM TECHNIQUE FOR SOLAR-PV APPLICATION.

24. Abd El Fattah El Atik and HananZaky, Ideal Expansion for some Nano Topological Structures, Journal of Computer and Mathematical Sciences, 9(11), (2018), 1639- 1652.

25. Ankit Gupta and RatnaDevSarma, A Note On Some Generalized Closure and Interior Operators in a Topological Space, Mathematics for Applications, 6, (2017), 11-20. 